\title{
Pattern formation in spatially heterogeneous Turing reaction-diffusion models
}

\author{
Karen Page $^{\mathrm{a}, *}$, Philip K. Maini ${ }^{\mathrm{b}}$, Nicholas A.M. Monk ${ }^{\mathrm{c}}$ \\ ${ }^{a}$ Bioinformatics Unit, Department of Computer Science, University College, London, Gower Street, London WC1E 6BT, UK \\ b Centre for Mathematical Biology, Mathematical Institute, 24-29 St. Giles', Oxford OX1 3LB, UK \\ ${ }^{\mathrm{c}}$ Centre for Bioinformatics and Computational Biology, Division of Genomic Medicine, University of Sheffield, \\ Royal Hallamshire Hospital, Sheffield S10 2JF, UK
}

Received 3 May 2002; received in revised form 14 October 2002; accepted 25 October 2002

Communicated by R.E. Goldstein

\begin{abstract}
The Turing reaction-diffusion model [Phil. Trans. R. Soc. 237 (1952) 37-72] for self-organised spatial pattern formation has been the subject of a great deal of study for the case of spatially homogeneous parameters. The case of parameters which vary spatially has received less attention. Here, we show that a simple step function heterogeneity in a kinetic parameter can lead to spatial pattern formation outside the classical Turing space parameter regime for patterning. This reduces the constraints on the model parameters, extending possible applications. Furthermore, it highlights the potential importance of boundaries during pattern formation.
\end{abstract}

() 2003 Elsevier Science B.V. All rights reserved.

Keywords: Pattern formation; Turing reaction-diffusion models; Self-organised spatial pattern

\section{Introduction}

In 1952, Turing [31] proposed a model for morphogenesis in which he hypothesised the existence of chemicals, termed morphogens, diffusing and interacting on a field of identical cells. He demonstrated that such a reaction-diffusion system in a closed spatially extended domain could, under appropriate parameter constraints, evolve into a spatially heterogeneous pattern, due to small fluctuations in chemical concentrations initiated by thermal noise alone. This phenomenon was termed diffusion-driven instability. He proposed that the strength of the chemical signal at each point in the domain would then determine the identity (differentiation fate) of the cell at that point. Thus, he was the first to formally define a morphogen and, some 50 years later, there is strong evidence that several such morphogens exist [29,32].

Since Turing's seminal paper, many reaction-diffusion models have been proposed and analysed, both mathematically and via numerical simulation (for reviews see, for example [8,12,22]). The vast majority of models studied involve only two chemical species since this is the simplest such system that exhibits the fundamental property of

* Corresponding author. Tel.: +44-20-7679-3683; fax: +44-20-7387-1397.

E-mail addresses: k.page@cs.ucl.ac.uk (K. Page), maini@maths.ox.ac.uk (P.K. Maini),n.monk@sheffield.ac.uk (N.A.M. Monk). 
diffusion-driven instability. The region of parameter space in which spatial patterns can form via this instability is called the Turing space.

The Turing systems that have been analysed fall into three categories: systems with simple kinetic functions which have been chosen because they give rise to patterns found in natural systems, systems whose kinetics (again usually simple) are based on hypothetical reactions, and those based on real reactions (see [19] for a review). Typical examples of these types of model were introduced by Gierer and Meinhardt [10], Schnakenberg [27] and Thomas [30].

In this paper, we consider as an illustrative example the Gierer-Meinhardt activator-inhibitor model, originally proposed to explain the regenerative properties of Hydra [10]. The equations describing the model are given in Appendix A.

Models such as these have been proposed to explain a wide variety of biological patterning processes, from the development of skin markings on the coats of mammals and fish [1,21] to the bifurcations of limbs [7] and the initiation of teeth in reptiles [17]. In these biological contexts, it is postulated that a reaction-diffusion system sets up a chemical "prepattern". Cells then respond to this pattern by differentiating only at sites where the chemical concentration exceeds a certain threshold. This notion was extended by Wolpert [33], who assumed that a much simpler spatially varying morphogen profile was set up by a source-sink mechanism conferring positional information on cells, which differentiate accordingly.

As an explanation for setting up a morphogenetic concentration field, the Turing model is still highly controversial, because strong evidence has yet to be found for actual reaction-diffusion morphogen prepatterns in vivo. However, Turing patterns have been demonstrated in real chemical systems [5].

Most theoretical work on Turing systems has considered models in which the kinetic parameters and diffusion rates are constant in space. However, in the context of morphogenesis, Turing himself recognised that "most of an organism, most of the time, is developing from one pattern to another, rather than from homogeneity into a pattern" [31]. Thus the reaction or diffusion rates of the chemical species may be modulated by preexisting spatial structure within the developing organism. Interest in the effects of domain patches on predator-prey models [34-36] lends further significance to the study of such reaction-diffusion systems with inhomogeneous parameters.

One specific form of spatial inhomogeneity that has recently been shown to play a particularly significant role in embryonic development is that of a sharp boundary within or between tissues. Such boundaries were predicted to act as organisers of pattern formation by Meinhardt [20], and recent studies have borne out this prediction in a wide range of developmental settings (reviewed in [15]). Developmental boundaries are often characterised by sharp discontinuities in gene expression. For example, in Drosophila, the boundaries between the dorsal and ventral compartments in the wing and eye imaginal discs separate fringe-expressing and nonexpressing cells [14]. The level of gene expression on either side of a boundary can, in principle, modulate both the kinetic and transport (for example, diffusion) parameters of a pattern-forming process. In the wing and eye discs in Drosophila, Fringe is believed to modulate the binding affinities between the receptor Notch and its ligands Delta and Serrate, resulting in localised gene expression at the Fringe-demarcated boundary [9,14,26]. Other factors could modulate the rate of transport of morphogens by changing the local properties of extra-cellular matrix or gap junctions, for example [4,6,28].

In general, the standard procedure for investigating model behaviour by finding spatially uniform steady states and determining their linear stability cannot easily be extended to systems with spatially varying parameters. One reason for this is that in most cases, a uniform steady state will not exist. An exception to this rule arises when the inhomogeneity occurs in the diffusion coefficient. This was exploited by Benson et al. [2], who explored the effect of a stepfunction in a diffusion coefficient, and by Hunding and Brøns [13], who provide a theoretical discussion of the solutions of a reaction-diffusion system with a small gradient in the diffusion rates.

Here we address instead systems with spatial inhomogeneity in a kinetic parameter so that the resultant system does not have a spatially uniform steady state. For simplicity we focus primarily on systems in one spatial dimension. In Section 2, we demonstrate numerically that the existence of a stepfunction in a kinetic parameter can lead to 
pattern formation outside the classical Turing parameter space. In Section 3, we show that a linear reaction-diffusion system can exhibit similar patterning. As this system is more analytically tractable, we can derive results on the form and stability of solutions. In Section 4, we apply these results to a linearised version of the Gierer-Meinhardt model system and show that the analytical solutions of this linearised system can be used to predict many features of the full nonlinear system. In Section 5, we show how pattern propagates away from the parameter discontinuity and how the system on each half of the domain is equivalent to a reaction-diffusion system with constant parameters on a domain with anomalous boundary conditions.

\section{Numerical solutions of the Gierer-Meinhardt model system with stepfunction kinetic parameter}

We nondimensionalise the Gierer-Meinhardt model equations [10] in a novel manner as detailed in Appendix A. The resultant equations are

$$
\begin{aligned}
& u_{t}=r\left(\frac{u^{2}}{v}+1\right)-\mu u+D_{u} u_{x x}, \quad v_{t}=r u^{2}-v v+D_{v} v_{x x} \quad \text { on }[0,1], \\
& u_{x}(0)=v_{x}(0)=u_{x}(1)=v_{x}(1),
\end{aligned}
$$

where $u(x, t)$ and $v(x, t)$ denote, respectively, the activator and inhibitor concentrations at spatial position $x$ and time $t$. This system has a spatially uniform steady state given by $u=(v+r) / \mu, v=r(v+r)^{2} / v \mu^{2}$ and there is a Turing bifurcation at $d \equiv D_{u} / D_{v}=(3 v+r-2 \sqrt{2 v(v+r)}) \mu / v(v+r)$.

We simulate these model equations numerically with example parameter values which lie outside the Turing space of (1), but within the range of stability of the uniform steady state to homogeneous perturbations. As expected no pattern evolves for the systems with constant parameters (parts (i) and (ii) of Fig. 1a). However, when we impose a stepfunction spatial variation in one of the parameters ( $\mu$ in this example), a stable spatially nonuniform pattern does form. This can be true even if the discontinuity in the parameter is small (Fig. 1b).

A key feature of these patterns is that they decay away from the centre of the domain. Thus if the domain were larger, the patterns would essentially be spatially confined. We find that this behaviour is also exhibited in linear reaction-diffusion systems. ${ }^{1}$ Fig. 2 shows the steady state pattern formed in a simulation of the example system:

$$
\left(\begin{array}{l}
u \\
v
\end{array}\right)_{t}=J\left(\begin{array}{l}
u \\
v
\end{array}\right)+D\left(\begin{array}{l}
u \\
v
\end{array}\right)_{x x}+\left(\begin{array}{c}
k_{1}(x) \\
k_{2}
\end{array}\right), \quad\left(\begin{array}{l}
u \\
v
\end{array}\right)_{x}=\left(\begin{array}{l}
0 \\
0
\end{array}\right) \quad \text { at } x=0,1,
$$

where

$$
J=\left(\begin{array}{ll}
j_{1} & j_{2} \\
j_{3} & j_{4}
\end{array}\right)
$$

and

$$
D=\left(\begin{array}{cc}
D_{u} & 0 \\
0 & D_{v}
\end{array}\right)
$$

Linear reaction-diffusion systems, such as (2), are not physically plausible as robust models for biological patterning since they can allow unbounded growth of the morphogen concentrations. However, the tractability of the linear problem and the similarities in the patterns formed motivate us to analyse this linear system to gain insight into

\footnotetext{
${ }^{1}$ Without spatially varying parameters, linear systems do not exhibit stable spatial patterns.
} 
(a) $\mathrm{i}$

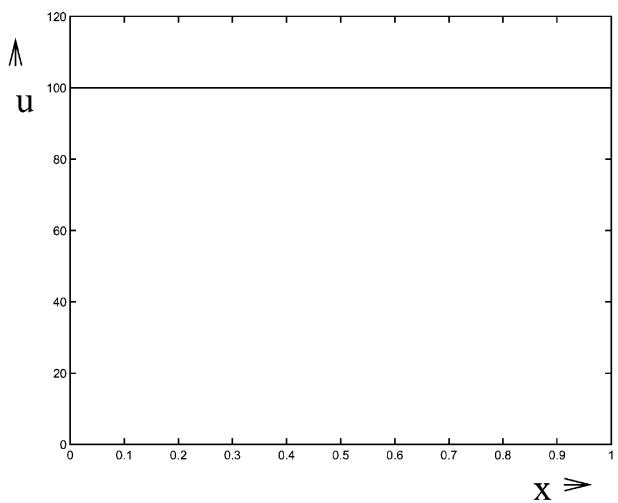

(b)

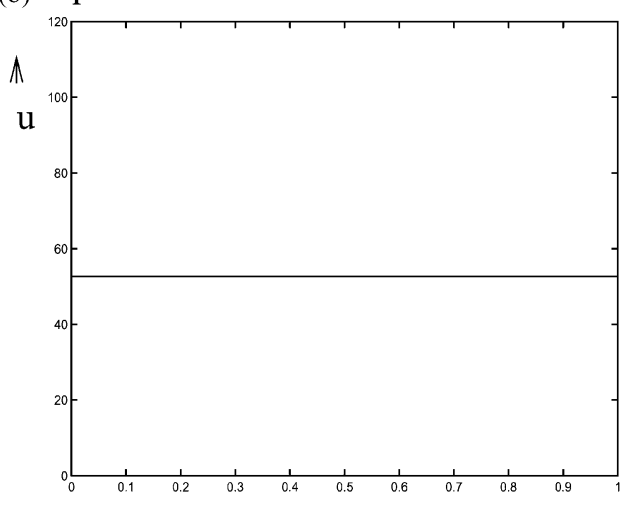

$x>$ ii
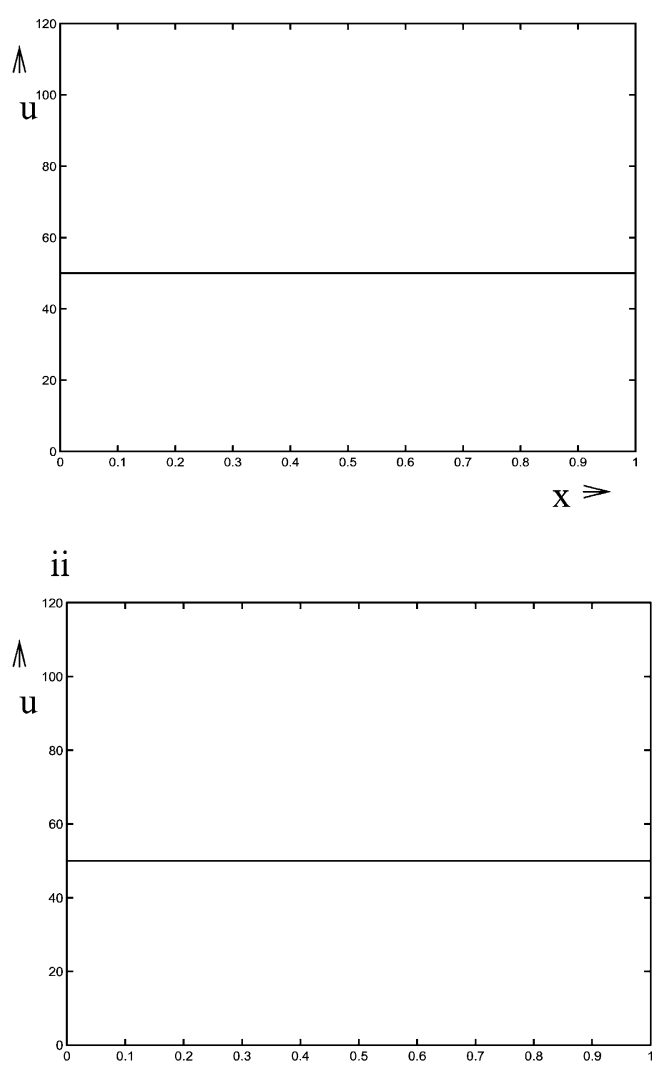

$\mathrm{x}>$ iii
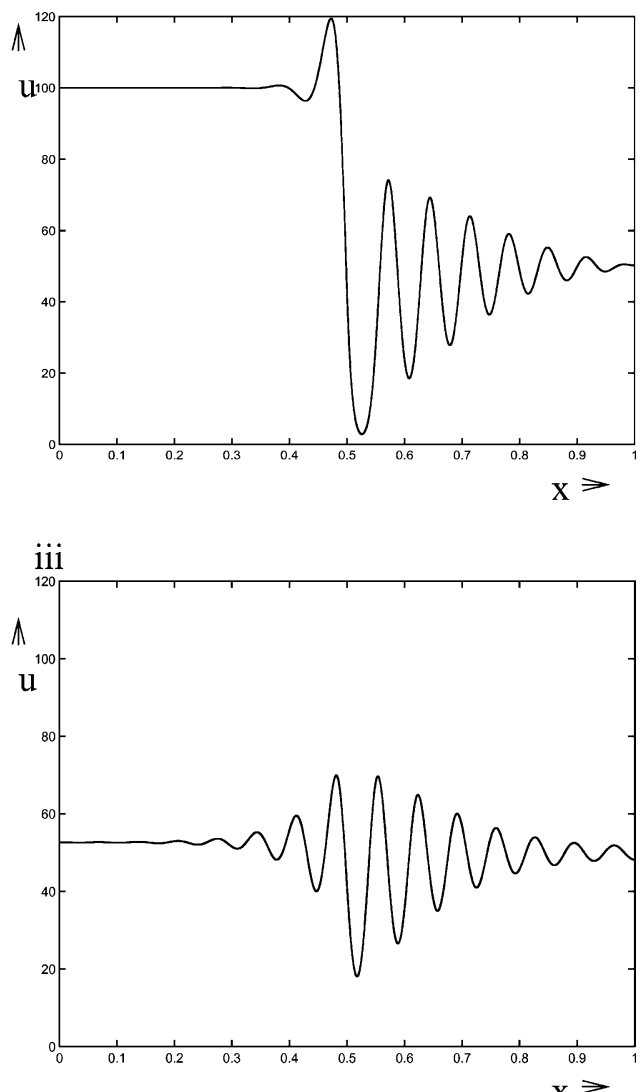

Fig. 1. Plots of the steady state activator concentration, $u(x)$, from simulations of the Gierer-Meinhardt equations (1) on the domain [0,1] with zero-flux boundary conditions, and parameters $D_{v}=0.027, d=0.0035, v=100.0, r=0.001$. (a) - (i) $\mu=1.0$; (ii) $\mu=2.0$; (iii) on the left-hand side of the domain, $\mu$ has the same value as in (i), while on the right-hand side $\mu$ has the same value as in (ii). (b) As above, except $\mu=1.9$ in (i). The steady state bifurcation value of $\mu$ in the system with constant parameters, with the other parameters as given, is 2.04. Thus all parameter values in the simulation results plotted lie outside the Turing space. (Numerical solver: NAG library routine: D03PCF (this is also used for the simulations in the other figures), number of discretisation points $=1001$, tolerance $=10 \mathrm{E}-07$, initial conditions $\pm 10 \%$ noise around the uniform steady states in (i) and (ii) and about the right-hand uniform steady state in (iii).) 


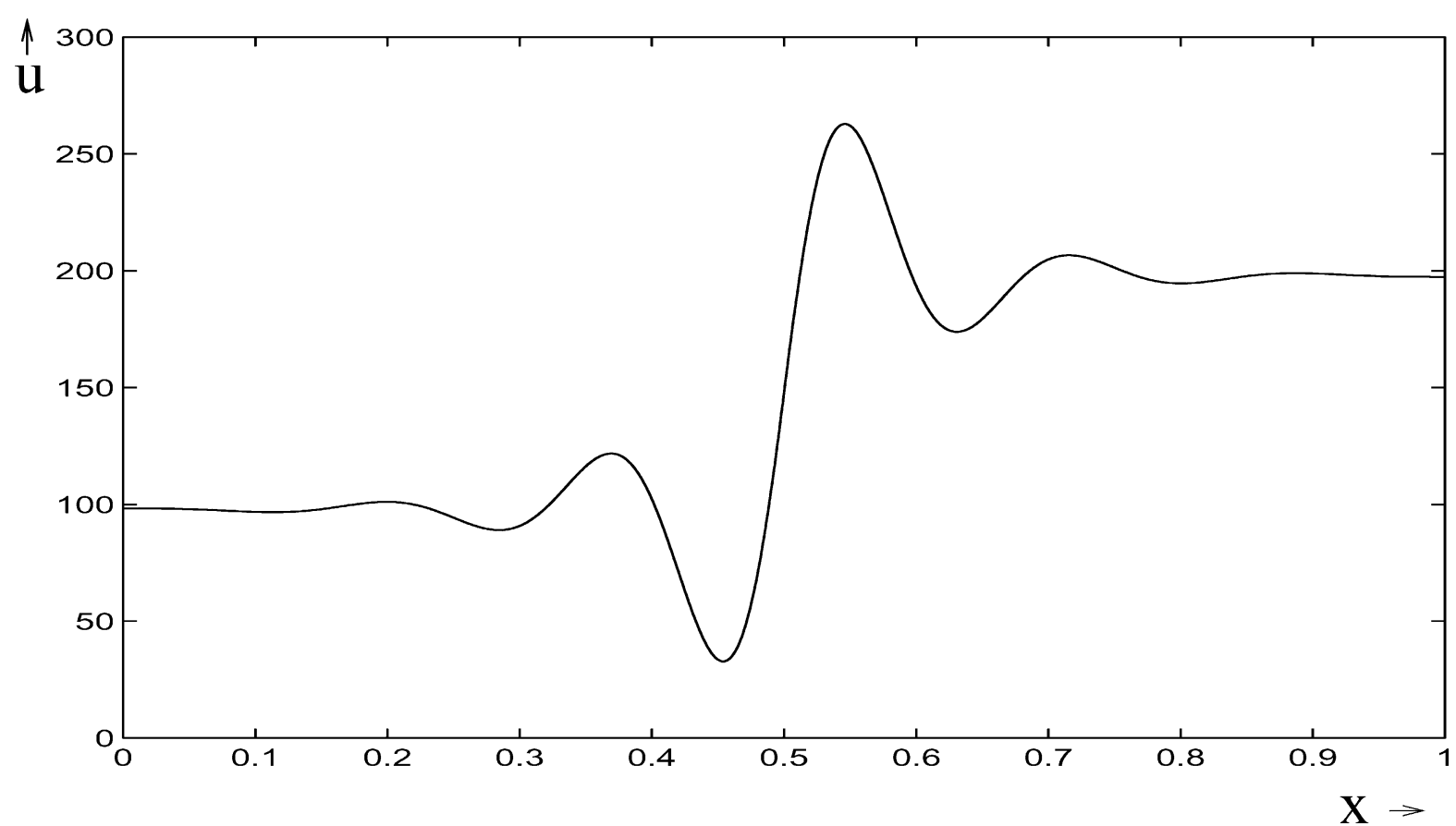

Fig. 2. Plot of the steady state activator concentration, $u(x)$, from simulations of the linear system (2), with a stepfunction in one of the parameters, on the domain $[0,1]$ with zero-flux boundary conditions, and parameters: $j_{1}=1.0, j_{2}=-22.0, j_{3}=5.0, j_{4}=-100.0$, $D_{v}=0.027, d=0.006, k_{2}=1.0$ and $k_{1}(x)=10.0$ if $x<1 / 2$ and $k_{1}(x)=20.0$ if $x>1 / 2$. See text for details. (Number of discretisation points $=1001$, tolerance $=10 \mathrm{E}-07$ and initial conditions $u=100.0$ and $v=50.0$.)

the patterning effects of spatially varying parameters in nonlinear models. We seek to understand how the presence of a boundary between different parameter values (or more generally a "prepattern" in the value of a parameter) can drive pattern formation and to determine what conditions must be satisfied in order for this to occur. We also attempt to explain the spatial localisation of the pattern.

\section{Analysis of the role of a stepfunction in a parameter in a linear reaction-diffusion model}

We consider the following linear nonhomogeneous system of reaction-diffusion equations:

$$
\left(\begin{array}{l}
u \\
v
\end{array}\right)_{t}=J\left(\begin{array}{l}
u \\
v
\end{array}\right)+D\left(\begin{array}{l}
u \\
v
\end{array}\right)_{x x}+\left(\begin{array}{c}
k_{1}(x) \\
k_{2}
\end{array}\right) \equiv \mathcal{L}\left(\begin{array}{l}
u \\
v
\end{array}\right)+\left(\begin{array}{c}
k_{1}(x) \\
k_{2}
\end{array}\right), \quad\left(\begin{array}{l}
u \\
v
\end{array}\right)_{x}=\left(\begin{array}{l}
0 \\
0
\end{array}\right) \quad \text { at } x=0,1,
$$

where

$$
J=\left(\begin{array}{ll}
j_{1} & j_{2} \\
j_{3} & j_{4}
\end{array}\right)
$$

is a matrix with constant entries and

$$
D=\left(\begin{array}{cc}
D_{u} & 0 \\
0 & D_{v}
\end{array}\right)
$$


is the matrix of diffusion coefficients. The matrix $J$ and the vector

$$
\underline{k}=\left(\begin{array}{c}
k_{1}(x) \\
k_{2}
\end{array}\right)
$$

are chosen such that the corresponding system with $\underline{k}$ replaced by the constant vector

$$
\left(\begin{array}{l}
k_{*} \\
k_{2}
\end{array}\right)
$$

has homogeneous steady state values of $u$ and $v$ which are positive, for any value of $k_{*}$ in the range of $k_{1}(x)$.

The steady state equations form a nonhomogeneous linear boundary value problem and have a unique solution except for values of the parameters for which $\mathcal{L}$ has a zero eigenvalue.

We consider the case where $k_{1}(x)$ takes a stepfunction form. We set $k_{1}(x)=a+b \mathcal{H}(x-\xi)$, where $a, b$ and $\xi$ are constants, $\xi \in(0,1)$, and $\mathcal{H}$ denotes the Heaviside function.

\subsection{Solution of the steady state equations}

Let

$$
\left(\begin{array}{l}
u_{0}(x) \\
v_{0}(x)
\end{array}\right)
$$

be the solution of the equation

$$
J\left(\begin{array}{l}
u \\
v
\end{array}\right)+\left(\begin{array}{c}
k_{1}(x) \\
k_{2}
\end{array}\right)=\left(\begin{array}{l}
0 \\
0
\end{array}\right) .
$$

This solution takes the form of a step function with values corresponding to the homogeneous steady states of the system with right-hand side and left-hand side parameters, respectively. Let

$$
\left(\begin{array}{l}
u \\
v
\end{array}\right)=\left(\begin{array}{l}
u_{0} \\
v_{0}
\end{array}\right)+\left(\begin{array}{l}
U \\
V
\end{array}\right) .
$$

The steady state equations become

$$
\begin{aligned}
& \left(\begin{array}{l}
U \\
V
\end{array}\right)_{x x}+J / D\left(\begin{array}{l}
U \\
V
\end{array}\right)=\left(\begin{array}{l}
0 \\
0
\end{array}\right), \quad x \in(0,1), \quad x \neq \xi \\
& \left(\begin{array}{l}
U \\
V
\end{array}\right)_{x}=\left(\begin{array}{l}
0 \\
0
\end{array}\right), \quad x=0,1, \quad\left(\begin{array}{l}
U \\
V
\end{array}\right)_{x}\left(x=\xi^{+}\right)-\left(\begin{array}{c}
U \\
V
\end{array}\right)_{x}\left(x=\xi^{-}\right)=\left(\begin{array}{l}
0 \\
0
\end{array}\right), \\
& \left(\begin{array}{l}
U \\
V
\end{array}\right)\left(x=\xi^{+}\right)-\left(\begin{array}{l}
U \\
V
\end{array}\right)\left(x=\xi^{-}\right)=\left(\begin{array}{l}
u_{0} \\
v_{0}
\end{array}\right)\left(x=\xi^{-}\right)-\left(\begin{array}{l}
u_{0} \\
v_{0}
\end{array}\right)\left(x=\xi^{+}\right),
\end{aligned}
$$

where $J / D$ denotes the matrix

$$
\left(\begin{array}{cc}
\frac{j_{1}}{D_{u}} & \frac{j_{2}}{D_{u}} \\
\frac{j_{3}}{D_{v}} & \frac{j_{4}}{D_{v}}
\end{array}\right) .
$$


The latter two equations come from the matching conditions at $x=\xi$, where we demand that $u, v, D_{u} u_{x}$ and $D_{v} v_{x}$ are continuous.

We diagonalise this system (following [2]) to obtain

$$
\begin{aligned}
& \left(U+s_{i} V\right)_{x x}+\lambda_{i}\left(U+s_{i} V\right)=0, \quad x \in(0, \xi) \cup(\xi, 1), \quad\left(U+s_{i} V\right)_{x}=0, \quad x=0,1, \\
& \left(U+s_{i} V\right)_{x}\left(x=\xi^{+}\right)-\left(U+s_{i} V\right)_{x}\left(x=\xi^{-}\right)=0, \\
& \left(U+s_{i} V\right)\left(x=\xi^{+}\right)-\left(U+s_{i} V\right)\left(x=\xi^{-}\right)=\left(u_{0}+s_{i} v_{0}\right)\left(x=\xi^{-}\right)-\left(u_{0}+s_{i} v_{0}\right)\left(x=\xi^{+}\right)
\end{aligned}
$$

for $i=1,2$, where we add $s_{i}$ times the second components of the vector equation (6) to the first components and pick $s_{i}$ such that

$$
\left(\begin{array}{c}
1 \\
-\frac{1}{s_{i}}
\end{array}\right)
$$

are eigenvectors of $J / D$. This choice leads to the decoupled form of the first equation, with $\lambda_{i}$ the eigenvalue corresponding to

$$
\left(\begin{array}{c}
1 \\
-\frac{1}{s_{3-i}}
\end{array}\right)
$$

The system of equations (7) has solution

$$
U+s_{i} V= \begin{cases}C_{i}^{L} \cos \sqrt{\lambda_{i}} x, & x<\xi \\ C_{i}^{R} \cos \sqrt{\lambda_{i}}(1-x), & x>\xi\end{cases}
$$

where $C_{i}^{L}$ and $C_{i}^{R}$ are constants determined by the matching conditions at $x=\xi$ :

$$
\left(\begin{array}{cc}
-\cos \sqrt{\lambda_{i}} \xi & \cos \sqrt{\lambda_{i}}(1-\xi) \\
\sqrt{\lambda_{i}} \sin \sqrt{\lambda_{i}} \xi & \sqrt{\lambda_{i}} \sin \sqrt{\lambda_{i}}(1-\xi)
\end{array}\right)\left(\begin{array}{c}
C_{i}^{L} \\
C_{i}^{R}
\end{array}\right)=\left(\begin{array}{c}
-\left(u_{0}^{J}+s_{i} v_{0}^{J}\right) \\
0
\end{array}\right),
$$

where $u_{0}^{J}=u_{0}\left(x=\xi^{+}\right)-u_{0}\left(x=\xi^{-}\right)$and similarly for $v_{0}$.

The matrix on the left-hand side of (9) has determinant $-\sqrt{\lambda_{i}} \sin \sqrt{\lambda_{i}}$ and so (9) has a unique solution, provided $\lambda_{i} \neq n^{2} \pi^{2}$. This will automatically be the case if the homogeneous steady state of the system without spatial variation in $k_{1}(x)$ is stable.

Thus, we have a unique solution for $U$ and $V$ provided $J / D$ has two distinct eigenvectors and $\lambda_{i} \neq n^{2} \pi^{2}$. The solution is given by

$$
\begin{aligned}
& U= \begin{cases}\frac{s_{1} C_{2}^{L} \cos \sqrt{\lambda_{2}} x-s_{2} C_{1}^{L} \cos \sqrt{\lambda_{1}} x}{s_{1}-s_{2}}, & x<\xi, \\
\frac{s_{1} C_{2}^{R} \cos \sqrt{\lambda_{2}}(1-x)-s_{2} C_{1}^{R} \cos \sqrt{\lambda_{1}}(1-x)}{s_{1}-s_{2}}, & x>\xi,\end{cases} \\
& V= \begin{cases}\frac{C_{1}^{L} \cos \sqrt{\lambda_{1}} x-C_{2}^{L} \cos \sqrt{\lambda_{2}} x}{s_{1}-s_{2}}, & x<\xi, \\
\frac{C_{1}^{R} \cos \sqrt{\lambda_{1}}(1-x)-C_{2}^{R} \cos \sqrt{\lambda_{2}}(1-x)}{s_{1}-s_{2}}, & x>\xi,\end{cases}
\end{aligned}
$$


where

$$
\left(\begin{array}{c}
C_{i}^{L} \\
C_{i}^{R}
\end{array}\right)=\frac{u_{0}^{J}+s_{i} v_{0}^{J}}{\sin \sqrt{\lambda_{i}}}\left(\begin{array}{c}
\sin \sqrt{\lambda_{i}}(1-\xi) \\
-\sin \sqrt{\lambda_{i}} \xi
\end{array}\right) .
$$

Thus, the solutions take the form $u=u_{0}\left(x=\xi^{-}\right)+K_{1} \cos \sqrt{\lambda_{1}} x+K_{2} \cos \sqrt{\lambda_{2}} x$ on the left-hand side of the domain and a similar form (with the same wavelengths) on the right-hand side. Hence, if the eigenvalues $\lambda_{1}$ and $\lambda_{2}$ are real and negative (this leads to a cosh form of solution), the solution has at most one turning point in each half of the domain. (This is given by the solution of $\sinh \left|\sqrt{\lambda_{2}}\right| x / \sinh \left|\sqrt{\lambda_{1}}\right| x=-\left(K_{1}\left|\sqrt{\lambda_{1}}\right|\right) /\left(K_{2}\left|\sqrt{\lambda_{2}}\right|\right)$ which can have at most one solution since the left-hand side is monotonic and the right-hand side is constant.) As we will see, these eigenvalues can be complex and hence a nonmonotonic pattern can form when the system with constant parameters only converges to a homogeneous steady state. When the eigenvalues are complex, the patterns decay away from the discontinuity and hence are spatially restricted.

\subsection{Properties of the solutions}

Theorem 1. If the homogeneous steady state of the ODE system with constant parameters is a stable node, then the stepfunction system with $d \equiv D_{u} / D_{v}=1$ has a steady state which has at most one turning point in $x<\xi$ and at most one turning point in $x>\xi$.

Proof. $d=1 \Rightarrow J / D=\left(1 / D_{v}\right) J$. So $J / D$ has real negative eigenvalues $\Leftrightarrow J$ has real negative eigenvalues $\Leftrightarrow$ the ODE system is a stable node. From the form of the solutions, we already have that the steady state solution of the stepfunction problem can have at most one turning point in each half if $J / D$ has real negative eigenvalues.

Now the trace of $J / D, \operatorname{tr}(J / D)$, is monotonic decreasing in $d$ from being unbounded and positive as $d \rightarrow 0$ to a negative value at $d=1$. Also, the determinant, $\Delta(J / D)$, is always positive, since, by simple algebra, it equals $\Delta(J) / D_{u} D_{v}$. Hence, as $d$ decreases from 1 to 0 , the system undergoes a Hopf bifurcation. If the homogeneous ODE system admits a stable node then there will be two changes of form at which the steady state of the stepfunction system goes from having a cosh form with at most one turning point in each half, to decaying oscillations to pure oscillatory as the eigenvalues go from real negative, to complex (initially negative real part then positive real part) to real positive. If the ODE system admits instead a stable spiral then there will only be one change of form as the steady state goes from decaying oscillations to pure oscillations.

We chose as an example, the system (3) with

$$
J=\left(\begin{array}{cc}
1.0 & -22.0 \\
5.0 & -100.0
\end{array}\right)
$$

and $D_{v}=0.027$ and

$$
\underline{k}= \begin{cases}\left(\begin{array}{c}
10.0 \\
1.0
\end{array}\right), & x<\frac{1}{2}, \\
\left(\begin{array}{c}
20.0 \\
1.0
\end{array}\right), & x>\frac{1}{2} .\end{cases}
$$


The homogeneous steady state of the corresponding ODE system (with $\underline{k}$ constant) is a stable node. The first change in form takes place at $d \equiv d_{1}=0.019$ and the second at $d \equiv d_{2}=0.0054$. Fig. 3 illustrates the form of the solution in between these points.

\subsection{Stability of solutions}

Consider a general linear nonhomogeneous reaction-diffusion system with spatially varying forcing (not necessarily a stepfunction) and zero-flux boundary conditions:

$$
\underline{u}_{t}=\mathcal{L} \underline{u}+\underline{f}(x), \quad \underline{u}_{x}=0 \quad \text { at } x=0,1 .
$$

Suppose $\underline{u}_{*}(x)$ is a steady state solution of (12) and consider its stability by adding a perturbation $\underline{\bar{u}}$, i.e. $\underline{u}=\underline{u}_{*}+\underline{\bar{u}}$. Then $\underline{\bar{u}}$ satisfies

$$
\underline{\bar{u}}_{t}=\mathcal{L} \underline{\bar{u}}, \quad \underline{\bar{u}}_{x}=0 \quad \text { at } x=0,1 .
$$

Thus, irrespective of the forcing, the stability of the unique (except in the cases mentioned earlier) solution of the nonhomogeneous problem depends on the stability of the zero steady state in the homogeneous problem. In particular, the patterned steady state of the two-species reaction-diffusion system with a stepfunction in a parameter, will lose stability, as $d$ is decreased, at the same point (critical value of $d$ ) as the homogeneous steady state of the corresponding system with a constant parameter loses stability. (We assume that, as in a standard two-species Turing system, $\operatorname{tr}(J)<0, \Delta(J)>0$ and $\left.j_{1}>0\right)$. The system becomes unstable to modes of the form $\cos n \pi x$.

Lemma 1. At the bifurcation of the $n \pi$ mode, $n^{2} \pi^{2}$ is an eigenvalue of $J / D$.

Proof. The mode with wavenumber $k$ starts to grow when one of the eigenvalues of $J-k^{2} D$ goes from having negative real part to having positive real part. Now $\operatorname{tr}\left(J-k^{2} D\right)<0 \forall D_{u}$ and $k$, so this happens when $\Delta\left(J-k^{2} D\right)=0$. But $\Delta\left(J-k^{2} D\right)=D_{u} D_{v} \Delta\left(J / D-k^{2} I\right)$, where $I$ is the identity matrix. So at the bifurcation $\Delta\left(J / D-k^{2} I\right)=0$, which implies that $k^{2}$ is an eigenvalue of $J / D$.

As $d$ is decreased, we note that, at the point at which the steady state loses stability, i.e. when the parameters enter the Turing space, $J / D$ has repeated eigenvalue $n^{2} \pi^{2}$ for some integer $n$, and just before this happens the matrix has complex eigenvalues close to this. Thus $\sin \sqrt{\lambda_{i}} \approx 0$ for $i=1,2$ and so from (9), the amplitude of the solution is large. Thus if the parameters are close to being in the Turing space, the amplitude of the decaying pattern in the stepfunction system is large.

Lemma 2. The unique (see above) steady state of the stepfunction system (2) loses stability after (or exactly when) it becomes purely oscillatory, as $d$ is decreased.

Proof. At the point of loss of stability $J / D$ has an eigenvalue $n^{2} \pi^{2}$ for some $n$, from Lemma 1 . From the discussion following Theorem 1 on the transitions in the form of the solution and the changes in the eigenvalues of $J / D$, this must occur when both eigenvalues of $J / D$ are positive, which is when the solution is pure oscillatory.

Lemma 3. If it were not for the constraint of the boundary conditions, the steady state would lose stability exactly when it becomes pure oscillatory. 

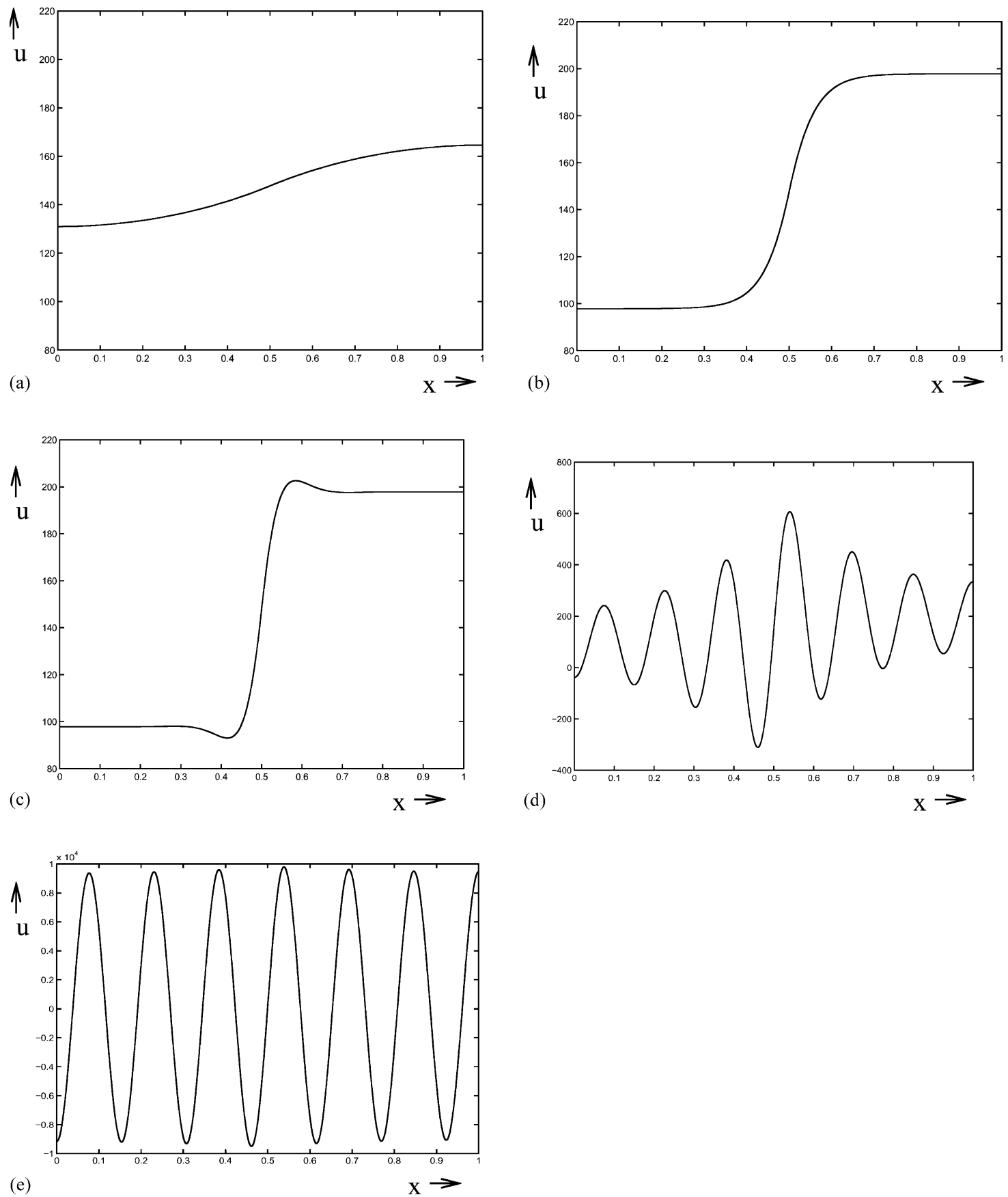

Fig. 3. Plot of the late-time steady state activator concentration, $u(x)$, from simulations of the same linear system as in Fig. 2, but with (a) $d=1.0$, (b) $d=0.020$, (c) $d=0.010$, (d) $d=0.0054$, (e) $d=0.005365$. Since the equations are linear, the solutions can become negative. Note that a transition in the solution profile occurs as $d$ changes from its value in (b) to that in (c) and a second transition occurs from (d) to (e). In (e) there is a very small window of parameter values before loss of stability. (Number of discretisation points $=1001$, tolerance $=10 \mathrm{E}-07$ and initial conditions $u=100.0$ and $v=50.0$.) 
Proof. Assuming all wavenumbers are allowed by the boundary conditions, loss of stability occurs when

$$
\Delta\left(J / D-k^{2} I\right)=0
$$

has a double root, $k=k_{\mathrm{c}}$. The fact that the root is repeated gives us

$$
k_{\mathrm{c}}^{2}=\frac{1}{2} \operatorname{tr}(J / D)
$$

and hence implies that $(1 / 2) \operatorname{tr}(J / D)$ is a repeated eigenvalue of $J / D$. This means that $\Delta(J / D)=((1 / 2) \operatorname{tr}(J / D))^{2}$ or equivalently $\operatorname{tr}(J / D)^{2}-4 \Delta(J / D)=0$. This corresponds to the point at which the eigenvalues of $J / D$ go from being complex to real (and positive since $k_{\mathrm{c}}^{2}$ must be positive).

\section{Numerical simulation of the Gierer-Meinhardt model and comparison with analytic results}

We found in the previous sections that we could not perform standard linear analysis on a general system of reaction-diffusion equations with a spatially varying parameter since there may not be a simple state (equivalent to the uniform steady state) around which perturbations can be everywhere small. However, we showed that when the inhomogeneity was a stepfunction then the key features of the pattern formation (that patterns could form when neither the left-hand nor the right-hand parameters were in the Turing space and that, in that case, the patterns decayed away from the discontinuity) were also exhibited in linear reaction-diffusion equations. It was possible to analyse these and hence gain insight into the patterning process in general. We look now to see if, in addition to this qualitative similarity between linear and nonlinear systems, there is a quantitative similarity. We numerically simulate the Gierer-Meinhardt model equations with a small added spatial inhomogeneity. For simplicity, we modify these equations by neglecting the $+r$ term in (1). We compare the solution with the analytical solution to the linear inhomogeneous system, which comes from linearising the homogeneous Gierer-Meinhardt equations about their uniform steady state and then adding in the spatial inhomogeneity term.

We consider the system of equations:

$$
u_{t}=r \frac{u^{2}}{v}-\mu u+d D_{v} u_{x x}+\Delta(x), \quad v_{t}=r u^{2}-v v+D_{v} v_{x x},
$$

where $\Delta(x)$ is the spatial inhomogeneity, and compare its numerical solution to the analytical solution of

$$
\left(\begin{array}{l}
u \\
v
\end{array}\right)_{t}=J\left(\begin{array}{l}
u \\
v
\end{array}\right)+D\left(\begin{array}{l}
u \\
v
\end{array}\right)_{x x}+\left(\begin{array}{c}
\Delta(x) \\
0
\end{array}\right),
$$

where

$$
J=\left(\begin{array}{cc}
\mu & -\frac{\mu^{2}}{r} \\
2 r \frac{v}{\mu} & -v
\end{array}\right)
$$

and

$$
D=\left(\begin{array}{cc}
d D_{v} & 0 \\
0 & D_{v}
\end{array}\right)
$$




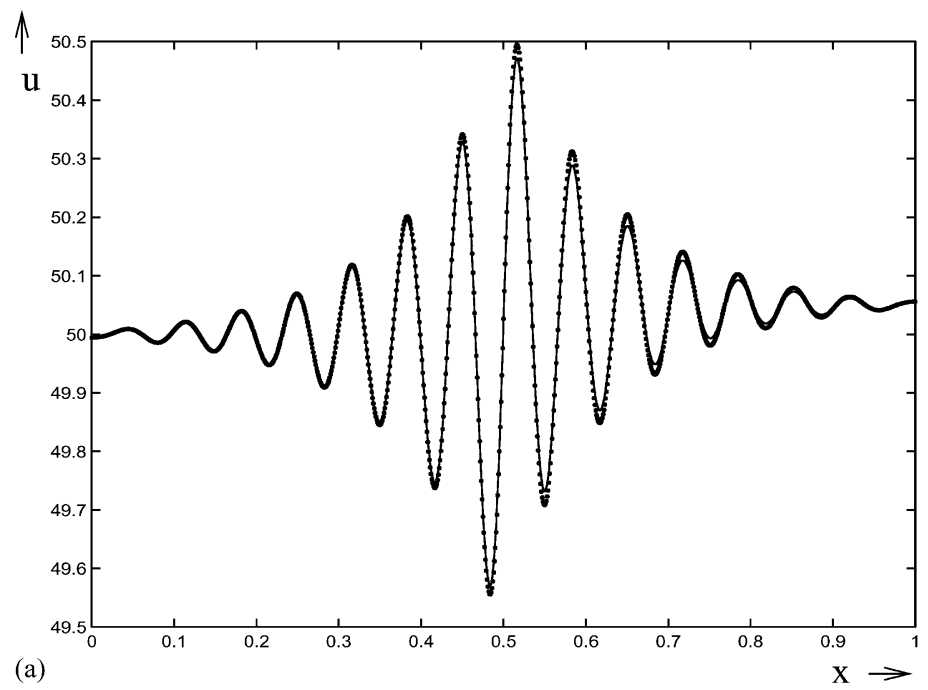

(a)

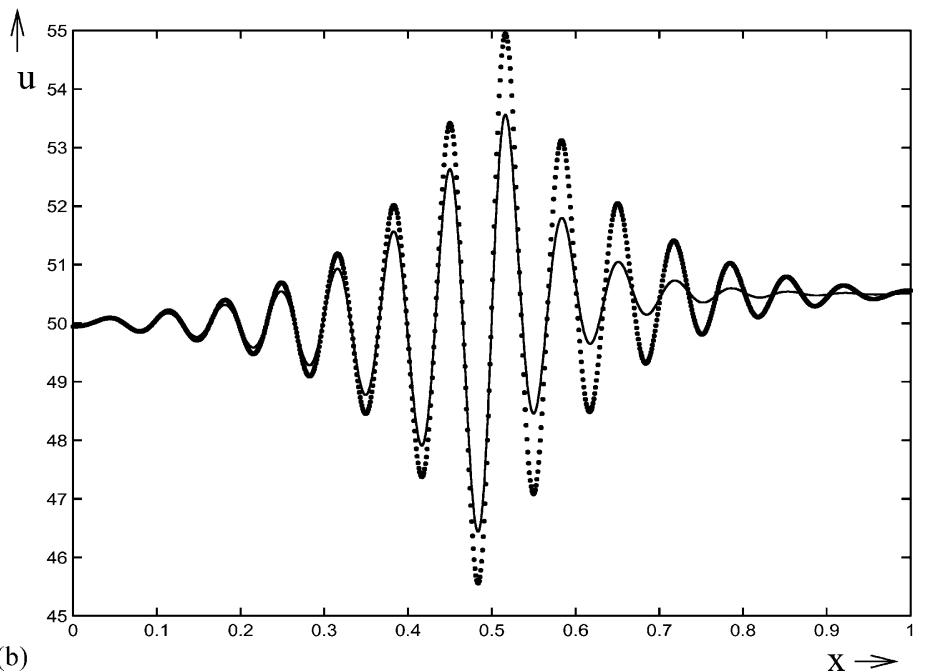

Fig. 4. Steady state solution of the Gierer-Meinhardt model with an added spatial inhomogeneity in the form of a stepfunction (solid line) and the analytical solution of the corresponding linear problem. Details and parameter values given in text: (a) a small stepfunction; (b) a larger stepfunction. (Number of discretisation points $=1001$, tolerance $=10 \mathrm{E}-07$ and initial conditions $\pm 10 \%$ noise around the uniform steady state of the left-hand parameters.)

Fig. 4 shows plots of the numerical solution of the nonlinear equation and the solution of the linear system for parameter values $v=100.0, \mu=2.0, d=0.0035, D_{v}=0.027$ and $r=0.001$. In (i),

$$
\Delta(x)= \begin{cases}0, & x<\frac{1}{2}, \\ 0.1, & x>\frac{1}{2} .\end{cases}
$$

From Eqs. (10) and (11), with the values of $\lambda_{1}, \lambda_{2}, s_{1}$ and $s_{2}$ determined by the eigenvalues and eigenvectors of $J / D$, we find that this yields the linear solution 


$$
u(x)= \begin{cases}50.0-(0.00573 \cos (93.76 x) \cosh (7.85 x)+0.0192 \sin (93.76 x) \sinh (7.85 x)), & x<\frac{1}{2}, \\ 50.05-(0.00573 \cos (93.76(1-x)) \cosh (7.85(1-x)) & \\ \quad+0.0192 \sin (93.76(1-x)) \sinh (7.85(1-x))), & x>\frac{1}{2} .\end{cases}
$$

In (ii),

$$
\Delta(x)= \begin{cases}0, & x<\frac{1}{2}, \\ 1.0, & x>\frac{1}{2},\end{cases}
$$

which yields solution

$$
u(x)= \begin{cases}50.0-(0.0573 \cos (93.76 x) \cosh (7.85 x)+0.192 \sin (93.76 x) \sinh (7.85 x)), & x<\frac{1}{2}, \\ 50.5-(0.0573 \cos (93.76(1-x)) \cosh (7.85(1-x)) & \\ \quad+0.192 \sin (93.76(1-x)) \sinh (7.85(1-x))), & x>\frac{1}{2} .\end{cases}
$$

We see from the figure that when the height of the stepfunction in $\Delta(x)$ is small $(0.1)$ the analytical linear solution is in very good agreement with the solution to the full nonlinear solution. However, for a large stepfunction discontinuity (1.0), although the analytical linear solution correctly predicts the number and position of the peaks, it overestimates their size.

\section{Propagation of the pattern away from a parameter discontinuity}

When there is a discontinuity in a parameter value of a reaction-diffusion system, the homogeneous steady state values of the variables on either side of the discontinuity will, in general, be different. If this is the case, then the discontinuity in the steady state values acts like a local perturbation and can trigger pattern formation if the homogeneous steady state on either side of the boundary is unstable. We postulate that, as with a local perturbation to the homogeneous steady state in a reaction-diffusion system with constant coefficients, the perturbation will cause the pattern to form as a wave propagating out from the disturbance with a speed and pattern wavenumber given by the marginal stability hypothesis [37]. The initial "perturbation" due to the discontinuity may not be small, but it is localised.

Fig. 5 shows the results of simulations of the Gierer-Meinhardt model system on a one-dimensional domain with a stepfunction in the activator decay rate in the middle of the domain at $x=0.5$. The other parameter values are chosen such that the homogeneous steady state is unstable to modes with a wavelength which is much smaller than the domain size, in order to minimise boundary effects. We see that, on either side, the pattern spreads out as a wave from the discontinuity. We calculate the approximate time taken for the pattern to reach a small threshold amplitude at $x=0.7,0.75,0.8$ and 0.85 and thus calculate the approximate speed of propagation of the wave between these points; we perform a similar operation on the left-hand side of the domain to calculate the wavespeed there. On the right-hand side of Fig. 5a, we get values of $t \approx 71.4,87.5,104.0$, 121.7 and hence the wave appears to move at roughly constant speed of around 0.0030. On the left-hand side of the domain, the wavespeed appears to take a roughly constant value of around 0.0027. These speeds compare with analytical values predicted by the marginal stability hypothesis of 0.0031 and 0.0027 , respectively (see [25]).

In Fig. 5b, the numerically calculated estimates of the wavespeed are 0.0075 for the right-hand side and 0.0028 for the left-hand side. These compare to analytical predictions of 0.0063 and 0.0027 , respectively [25]. Hence all 
(a) $\mathrm{i}$
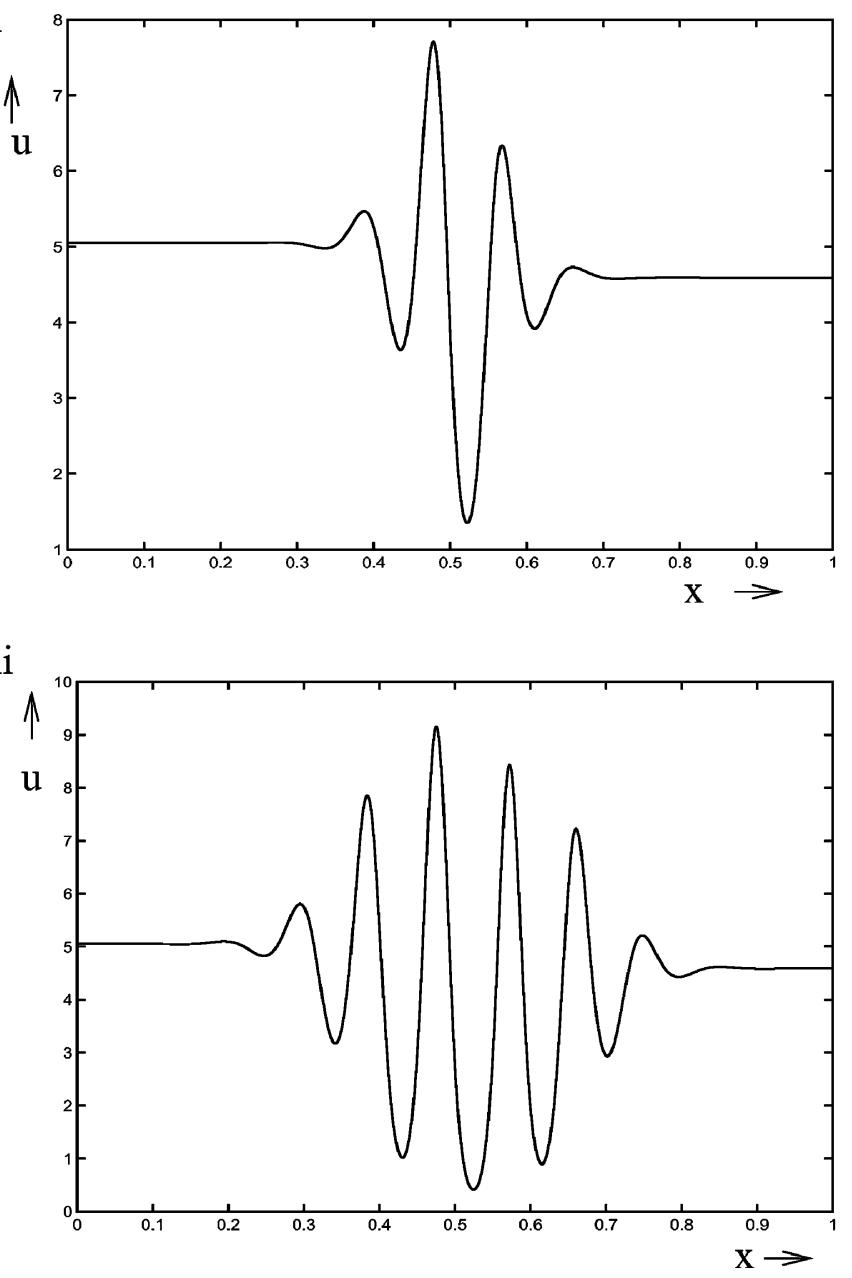

(b) $\mathrm{i}$

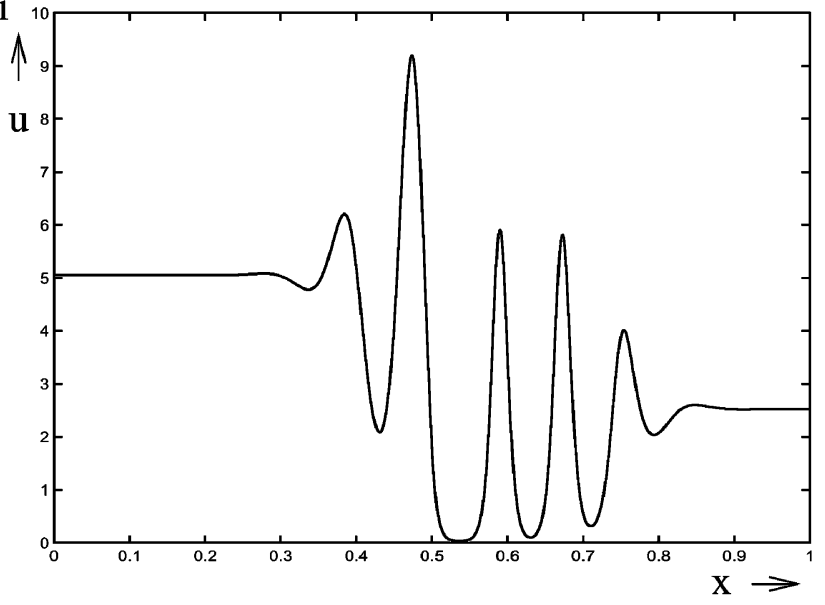

ii

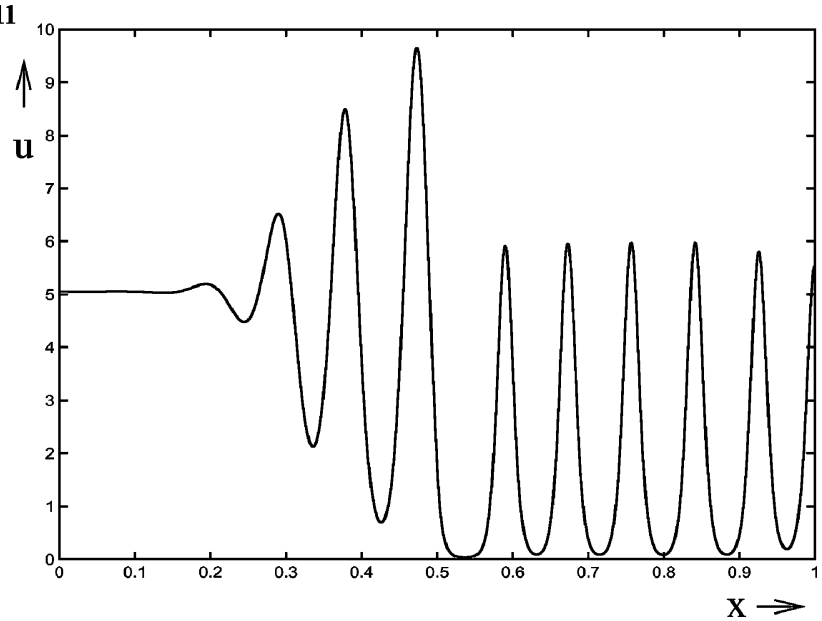

Fig. 5. Plot of the activator profiles at various times in simulations of the Gierer-Meinhardt model equations with a stepfunction in the activator decay rate. The domain is [0,1] (zero-flux boundary conditions) and the parameters are $D_{v}=0.000675, v=1.0, r=0.01$ and $d=0.02$. (a) $\mu=0.2$ on left-hand side, $\mu=0.22$ on right-hand side; (b) $\mu=0.2$ on left-hand side, $\mu=0.4$ on right-hand side (the initial conditions take the form of stepfunctions with homogeneous steady state values of the problem with constant coefficients on each half), in (i) $t=50$, (ii) $t=100$, (iii) $t=1000$ and in (iv) we show a greyscale plot of the activator concentration in space and time (lighter colour indicates higher concentration). 
iii
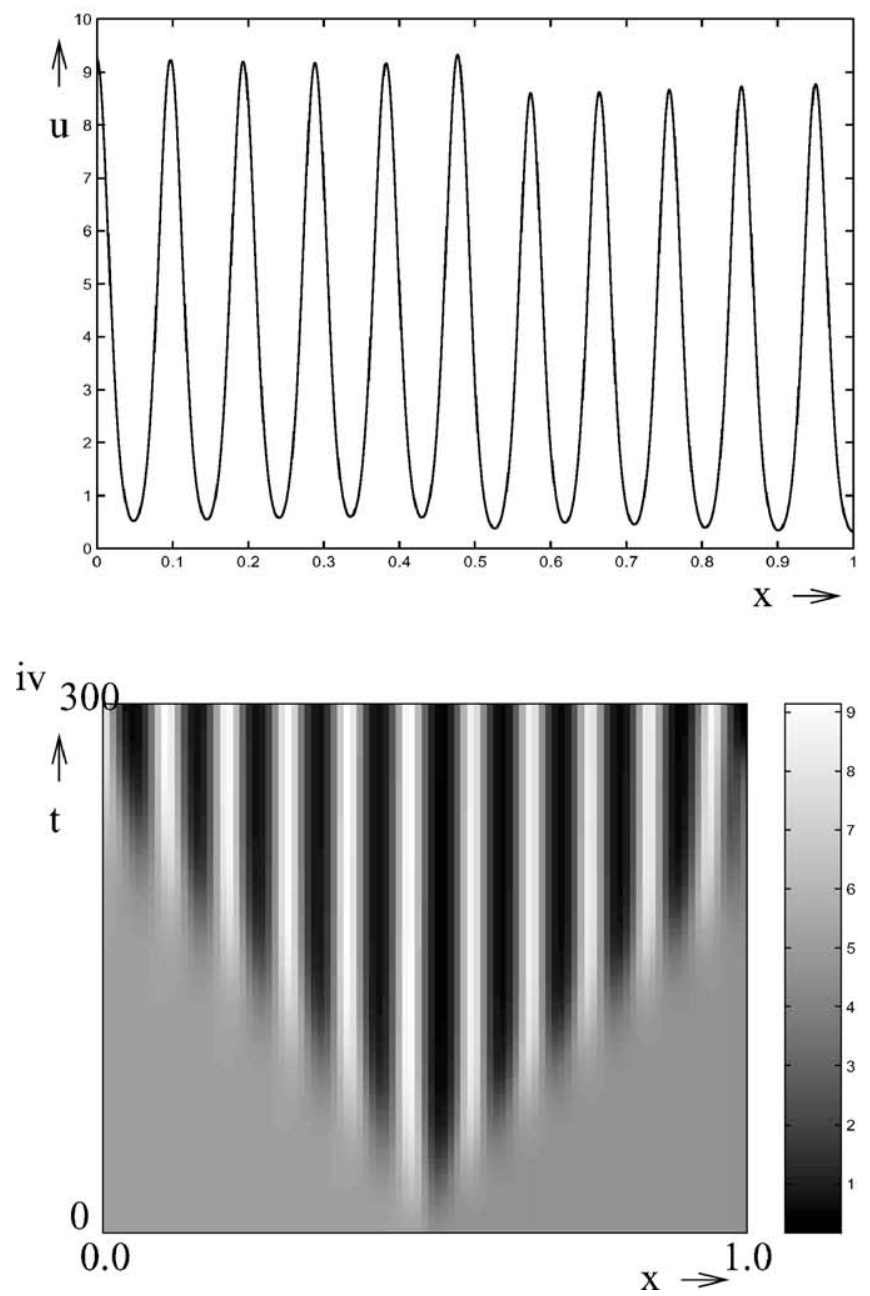

iii
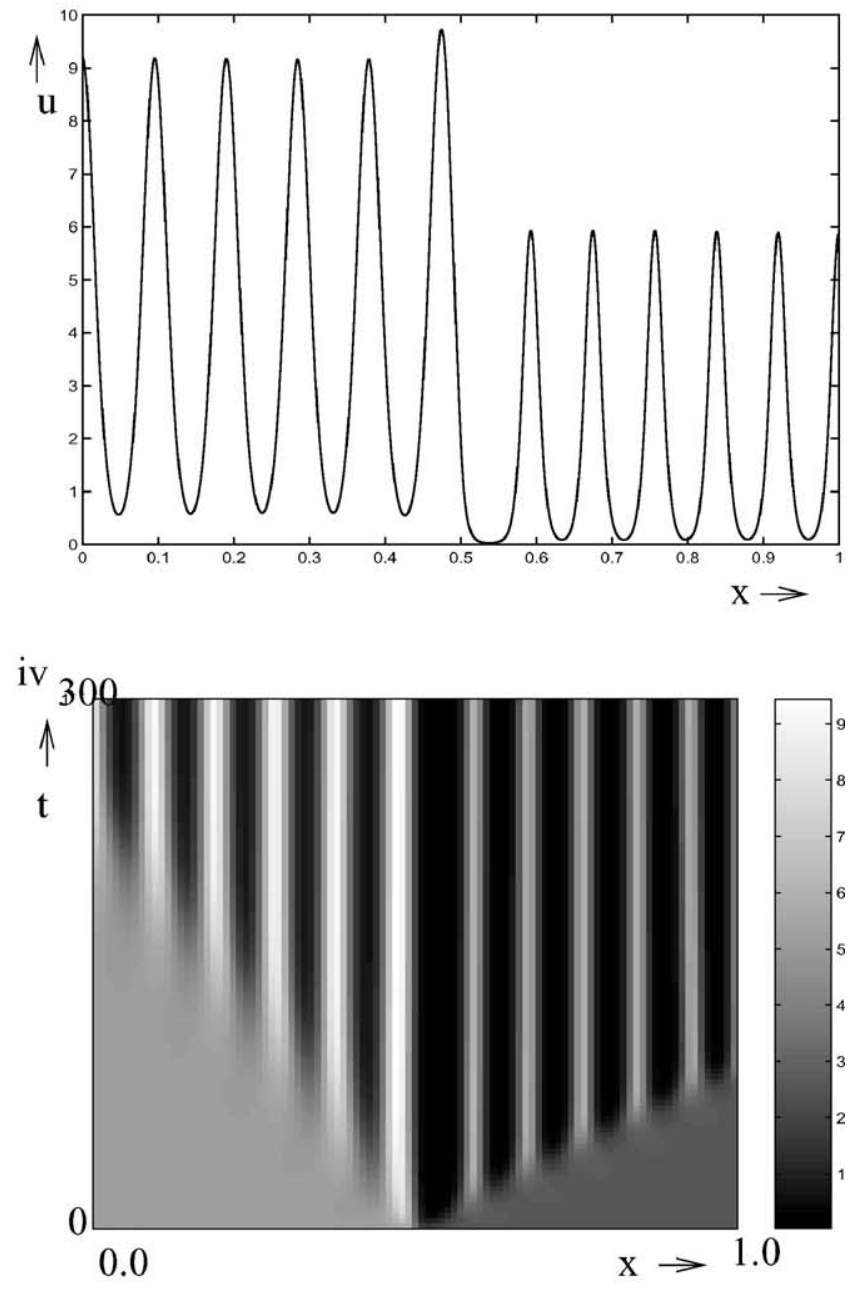

7
0
0
0
0
0
0
0
0
0
0
0
0
0
0
0
0
0
0
0
0
0

Fig. 5. (Continued). 
the predictions are good approximations to the wavespeed except for the one for the right-hand side of (b). This is possibly because the wave in this case is faster and so more susceptible to boundary effects; it seems to speed up as it reaches the boundary. The pattern left behind the wave is roughly periodic and the wavelength is consistent with the marginal stability hypothesis which predicts roughly 5.5 peaks on either side of the domain in both (a) and (b). On the right-hand side of (b), the prediction is actually 5.77, but in the other cases 5.5 is the nearest half number to the prediction. In the case of the large stepfunction in the parameter, the first peak is somewhat higher and wider than the others, but this irregularity appears localised (the left-hand side steady state patterns are very similar, (b) having a slightly higher first peak and the other peaks being slightly further from the discontinuity). Once the wave has begun to propagate, the leading edge perturbation is always infinitesimal and so the system behaves as if the perturbation were small and local. We can clearly see the approximately constant speed of propagation in a greyscale plot of the activator concentration in space and time, as shown in part (iv) of Fig. 5a and b.

\section{Analysis of parameter discontinuity in a general reaction-diffusion model}

We now attempt to gain analytical insight into how the pattern forms in a general reaction-diffusion system with a discontinuity in a parameter.

We consider the system

$$
u_{t}=f(u, v ; \alpha(x))+D_{u} u_{x x}, \quad v_{t}=g(u, v ; \alpha(x))+D_{v} v_{x x},
$$

with zero-flux boundary conditions on the domain $[0, L]$, where $\alpha$ is a parameter which takes the following form:

$$
\alpha(x)= \begin{cases}\alpha_{0}-\epsilon, & 0 \leq x<\frac{L}{2}, \\ \alpha_{0}+\epsilon, & \frac{L}{2}<x \leq L,\end{cases}
$$

where $\epsilon$ is a small parameter. We assume that when $\epsilon=0$, i.e. when $\alpha$ is constant, (20) admits a homogeneous steady state $(u, v)=\left(u_{0}, v_{0}\right)$. We also assume that this state is linearly stable (we choose parameters outside the Turing space). We consider a perturbation solution of (20) for $\epsilon \neq 0$ :

$$
\left(\begin{array}{l}
u \\
v
\end{array}\right)=\left(\begin{array}{l}
u_{0} \\
v_{0}
\end{array}\right)+\epsilon\left(\begin{array}{l}
u_{1}(x, t) \\
v_{1}(x, t)
\end{array}\right)+\epsilon^{2}\left(\begin{array}{l}
u_{2}(x, t) \\
v_{2}(x, t)
\end{array}\right)+\cdots .
$$

To $\mathrm{O}(1)$, we simply have $f\left(u_{0}, v_{0} ; \alpha_{0}\right)=g\left(u_{0}, v_{0} ; \alpha_{0}\right)$. Collecting terms of $\mathrm{O}(\epsilon)$, we obtain

$$
\left(\begin{array}{l}
u_{1} \\
v_{1}
\end{array}\right)_{t}=J\left(\begin{array}{l}
u_{1} \\
v_{1}
\end{array}\right)+D\left(\begin{array}{l}
u_{1} \\
v_{1}
\end{array}\right)_{x x}+\operatorname{sgn}\left(x-\frac{L}{2}\right)\left(\begin{array}{l}
f_{\alpha}\left(u_{0}, v_{0}, \alpha_{0}\right) \\
g_{\alpha}\left(u_{0}, v_{0}, \alpha_{0}\right)
\end{array}\right)
$$

and $\left(u_{1}\right)_{x}=\left(v_{1}\right)_{x}=0$ at $x=0, L$, where $J$ is the Jacobian matrix at $\left(u_{0}, v_{0}\right)$ for the system with $\alpha=\alpha_{0}$, $D$ the matrix of diffusion coefficients and $f_{\alpha}$ and $g_{\alpha}$ the functions of $(u, v)$ obtained by differentiating $f$ and $g$ with respect to $\alpha$. Now $f_{\alpha}\left(u_{0}, v_{0}, \alpha_{0}\right)$ and $g_{\alpha}\left(u_{0}, v_{0}, \alpha_{0}\right)$ are constant in space and time, so (23) corresponds 
to a linear equation in $\left(u_{1}, v_{1}\right)$, with a spatially varying term which is simply added to the linear homogeneous terms.

The above analysis would hold likewise for any scalar boundary conditions (conditions of the form $\delta \underline{u}_{x}+\beta \underline{u}=\underline{\gamma}$ at $x=0,1)$. If we considered a system in which the case $\epsilon=0$ had a stable steady solution which was patterned rather than homogeneous, then the analysis would hold except that both $J$ and

$$
\left(\begin{array}{l}
f_{\alpha}\left(u_{0}, v_{0}, \alpha_{0}\right) \\
g_{\alpha}\left(u_{0}, v_{0}, \alpha_{0}\right)
\end{array}\right)
$$

would vary in space.

It is clear that (23) is invariant under the transformation $x \rightarrow L-x, u_{1} \rightarrow-u_{1}, v_{1} \rightarrow-v_{1}, t \rightarrow t$. Thus if the initial conditions are also antisymmetric in $x$, i.e. $u_{1}(x, 0)=-u_{1}(L-x, 0)$, then the solution will always be antisymmetric. In particular $u_{1}(L / 2)=-u_{1}(L / 2)$ which implies $u_{1}(L / 2)=0 \forall t$. Hence, if the initial conditions in (23) are antisymmetric, then the problem is the same on each half of the domain as the corresponding problem in which the inhomogeneous term is a constant and the boundary conditions are $u_{1}, v_{1}=0$ at $x=L / 2$, and zero flux at the other end. On the left-hand side of the domain, the constant takes value

$$
-\left(\begin{array}{l}
f_{\alpha}\left(u_{0}, v_{0}, \alpha_{0}\right) \\
g_{\alpha}\left(u_{0}, v_{0}, \alpha_{0}\right)
\end{array}\right)
$$

and on the right-hand side of the domain, the constant takes value

$$
+\left(\begin{array}{c}
f_{\alpha}\left(u_{0}, v_{0}, \alpha_{0}\right) \\
g_{\alpha}\left(u_{0}, v_{0}, \alpha_{0}\right)
\end{array}\right) .
$$

The solution of this equation is equivalent (up to the addition of a constant) to the solution of the homogeneous equation (no constant added) with a zero-flux boundary condition and an anomalous fixed boundary condition (the solution is fixed to a value which is not the homogeneous steady state value at one of the boundaries).

In Fig. 6, we show for comparison the results of a simulation of the Gierer-Meinhardt model with constant parameters and an anomalous boundary condition. As predicted by this analysis, we see a similar pattern of decaying oscillations as found in the corresponding stepfunction system (compare Fig. 6 with part (iii) of Fig. 1a).

We can solve Eq. (20) by considering the solution as the sum of the steady state solution $\underline{u} \equiv(\bar{u}(x), \bar{v}(x))$ (which we showed how to find in Section 3) and a correction term $\tilde{u} \equiv(\tilde{u}(x, t), \tilde{v}(x, t))$. If the initial conditions on $\underline{u} \equiv(u, v)$ are $\underline{u}(x, 0)=f(x)$, then $\underline{\tilde{u}}$ satisfies

$$
\underline{\tilde{u}}_{t}=J \underline{\tilde{u}}+D \underline{\tilde{u}}_{x x}
$$

with $\underline{\tilde{u}}=0$ at $x=0, L$ and $\underline{\tilde{u}}(x, 0)=\underline{f}(x)-\underline{\bar{u}}(x)$. This is a linear homogeneous system of equations with scalar boundary conditions and so can be solved as a sum of eigenfunctions (in this case cosine modes). The initial conditions determine the initial amplitudes of these eigenfunction modes. The amplitudes decay in time to zero. It is likely that $f$ will take the form of a constant (with value corresponding to the uniform steady state values of the species for the system with parameters of either the left-hand side or right-hand side) or else a stepfunction (with the steady state values of the left-hand system on the left-hand side and of the right-hand system on the right-hand side). Either way, the initial symmetrical component is simply a constant. The decay of the constant mode is large (corresponding to the modulus of the real part of the eigenvalue of $J$ with the negative real part of small modulus) and hence the symmetric (constant) component rapidly disappears. Some of the odd cosine modes present in $\underline{f}-\underline{\tilde{u}}$ do not decay so quickly and hence the convergence to a steady state pattern happens on a longer timescale than convergence to an antisymmetric solution. It is not obvious from this form of the solution that $\underline{u}$ converges rapidly 


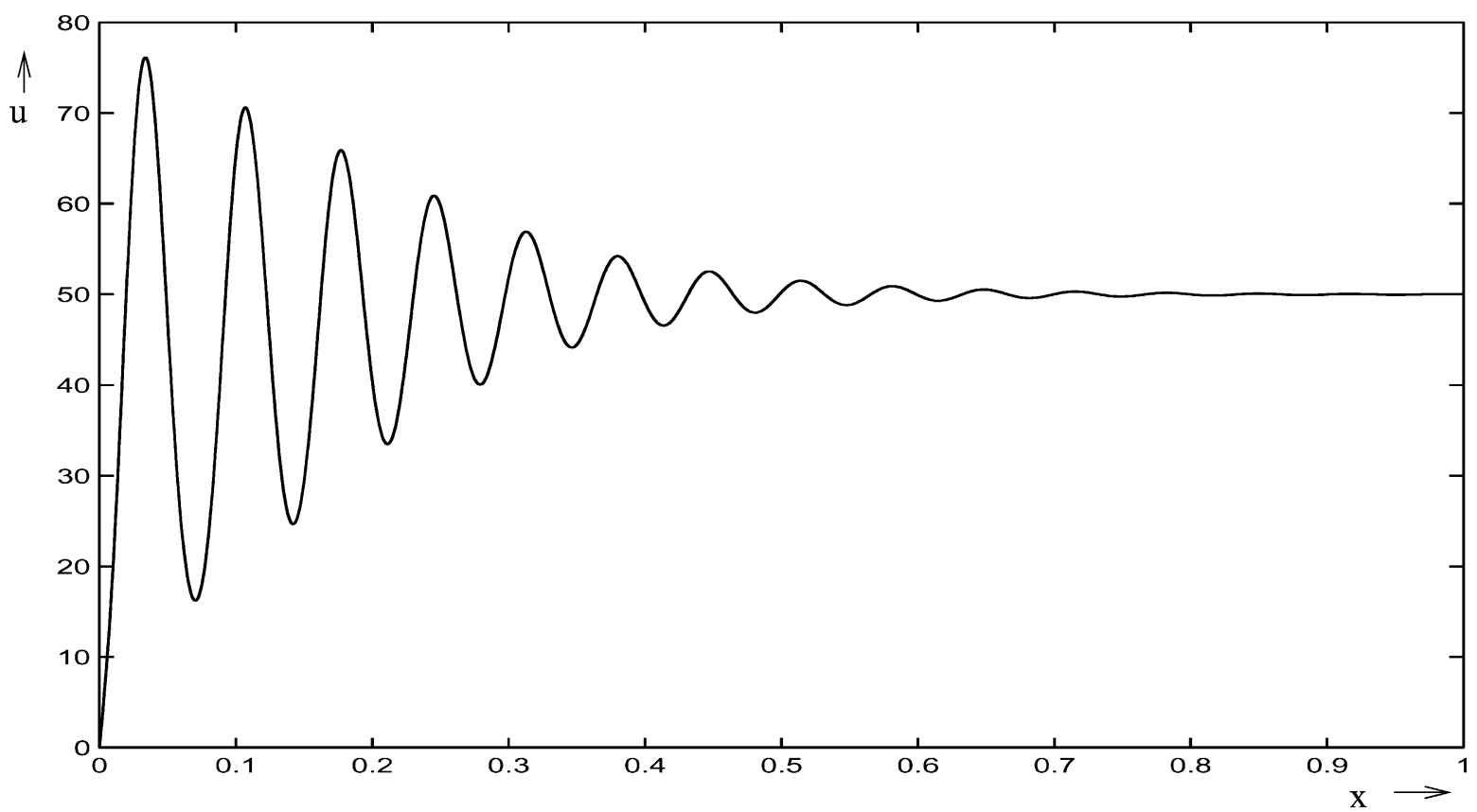

Fig. 6. Having one zero-flux boundary condition and one fixed boundary condition (at a level which is not the homogeneous steady state) leads to a pattern of decaying oscillations similar to those in part (iii) of Fig. 1a and b. Parameter values are $v=100.0, \mu=2.0, r=0.001, D_{v}=0.027$ and $d=0.0035$. We impose zero-flux boundary conditions at the right-hand boundary and fixed values $u=0$ and $v=0.01$ at the left-hand boundary. (Number of discretisation points $=1001$, tolerance $=1.0 \mathrm{E}-07$ and initial conditions are $\pm 10 \%$ noise around the uniform steady state of the system with zero-flux boundary conditions.)

to its final value near the edges of the domain, that the solution rapidly becomes relatively flat away from the stepfunction in the centre of the domain and that the oscillations of the steady state solution propagate outwards from the middle, as found in numerical simulations. To show this would take further analytical investigation.

In Fig. 7, we show the way in which (a) $u$ and (b) $\tilde{u}$ develop in time, in the linear system on the domain $[0,1]$ with

$$
\begin{aligned}
& J=\left(\begin{array}{cc}
1.0 & -22.0 \\
5.0 & -100.0
\end{array}\right), \\
& D=\left(\begin{array}{cc}
3.78 \times 10^{-5} & 0.0 \\
0.0 & 6.75 \times 10^{-3}
\end{array}\right), \\
& \underline{k}=\left\{\begin{array}{c}
\left(\begin{array}{c}
10.0 \\
1.0
\end{array}\right), \quad x<\frac{1}{2}, \\
\left(\begin{array}{c}
20.0 \\
1.0
\end{array}\right), \quad x>\frac{1}{2},
\end{array}\right.
\end{aligned}
$$

and initial conditions $u=100.0, v=5.0$, which are roughly the steady state values on the left-hand side (the initial conditions are symmetric). In field (i), we show the early time behaviour; the dotted lines show the solutions at $t=10$ and the solid lines show the solutions at $t=50$. We can see that the value of $u$ near the right-hand 

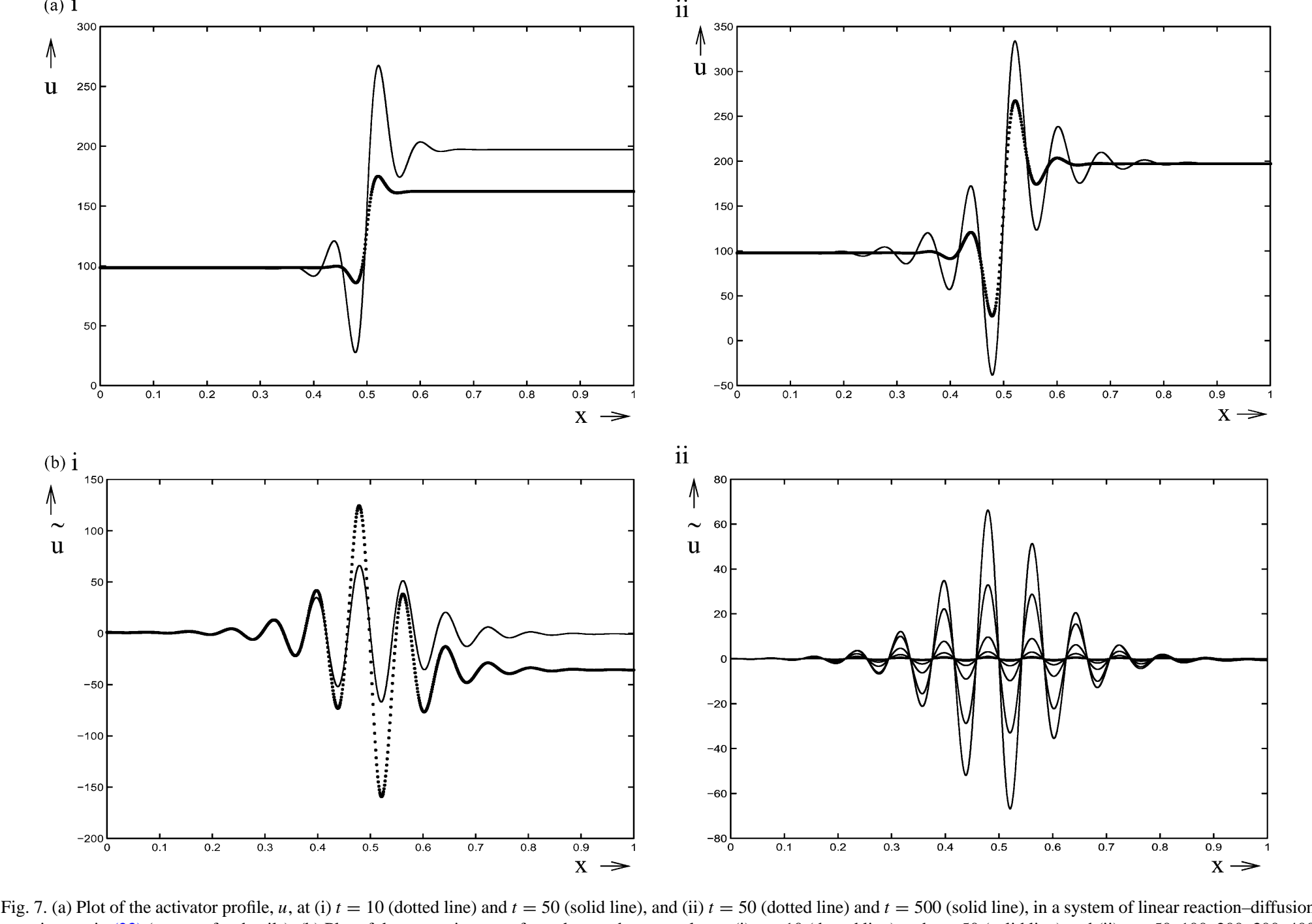
boundary rapidly converges to near its steady state value at that boundary and the value of $\tilde{u}$ rapidly converges to zero near both boundaries. In field (ii), we show the later time behaviour. Part (ii) of Fig. 7a shows the solution for $u$ at $t=50$ (dotted line) and $t=500$ (solid line). It shows growth and spreading out of the pattern from the centre of the domain. Part (ii) of Fig. 7b shows the solution for $\tilde{u}$ at $t=50,100,200,300,400$ and 500. It indicates a fairly uniform decay.

\section{Discussion}

We have shown that a stepfunction in a parameter of a reaction-diffusion system can lead to a complex spatial pattern when constant parameters at the level of either side of the discontinuity would not. Stable large amplitude patterns can form even if the discontinuity in the parameter is small. Thus, inhomogeneities in parameters can lead to an extension of the classical Turing parameter space. Furthermore, we have shown that the resulting patterns are spatially localised around the parameter discontinuity. Spatial localisation in the pattern can also occur in reaction-diffusion systems with mixed boundary conditions [18]. For the case of a small stepfunction, we have demonstrated that such systems are formally equivalent to one-half of the domain in the corresponding system with a stepfunction in a parameter. We have also shown that patterns tend to propagate outwards from a parameter discontinuity with a speed and pattern wavelength given approximately by the marginal stability hypothesis.

We have gained analytical insight into the features of the stable patterns resulting from stepfunction discontinuities by studying a linear reaction-diffusion model. Mathematical analysis of the stepfunction problem in this system shows that the steady state has at most one turning point in each half of the domain if the steady state of the corresponding ordinary differential equation (ODE) is a stable node. If the steady state of the ODE system is a stable spiral, then the solution of the stepfunction problem exhibits decaying oscillations. Finally, if it were not for the constraints of the boundary conditions, then the solution would lose stability exactly when it became pure oscillatory. Using numerical simulation, we have shown that a suitable linearised system exhibits many of the same features as the full nonlinear Gierer-Meinhardt system and hence that the analytical results obtained can approximately predict key features of the nonlinear system.

Spatial patterns triggered by stepfunction discontinuities in a parameter could have important implications for the role of boundaries in pattern formation during development. There are many biological systems in which patterning occurs in a hierarchical fashion (see, for example [1,11,20,23,24]). A specific example of hierarchical patterning results from the establishment of sharp boundaries within developing tissues, separating populations of cells that differ in the level of expression of one or more genes (reviewed in [15]). As predicted by Meinhardt [20], such boundaries play significant roles in the patterning of the surrounding tissue. In many cases, such as in Drosophila eye imaginal discs and developing appendages in Drosophila and vertebrates, specific gene expression and subsequent cell differentiation occur at or around such boundaries $[3,9,14,16,26]$.

Our results show that boundaries separating cells with different gene expression profiles can have significant implications for patterning mechanisms operating in the tissue containing the boundary. If one or more of the parameters of the patterning mechanism were modulated by the differentially expressed genes, then we have shown for the case of a reaction-diffusion mechanism that spatial patterns can be generated even when the parameters in either region are outside the range that supports pattern formation. This novel result of patterning outside of the Turing space provides a specific illustration of the importance of hierarchies of patterning in development [11], i.e. it demonstrates the influence of existing inhomogeneities in the system not only on the specific form of new patterns, but on their ability to form at all.

In this paper, we have restricted our attention to investigating the effect of a stepfunction in a parameter on pattern formation via reaction-diffusion in one dimension. Numerical simulations on a two-dimensional domain show that 
a prepattern in a control species can lead to patterns of spatially varying wavelength, for example patterns of large and small spots, resembling those seen on the head of the lizard Cyrtodactylus fedtschenkoi, or novel target-like patterns. Also, a system which would normally give rise to spots can instead produce striped patterns (Page, Maini and Monk, in preparation). Hence, in addition to widening the range of possible patterns, prepatterns in a parameter can also select patterns in the reaction-diffusion system. This provides further confirmation that, at least in some cases, hierarchical patterning can result in more robust patterning than can de novo patterning (as suggested in [11]).

\section{Acknowledgements}

KMP would like to thank the Joint Research Councils (EPSRC, BBSRC and MRC-grant no. GR/R47455) for a lectureship in bioinformatics. PKM would like to thank the Issac Newton Institute for Mathematical Sciences, University of Cambridge, for a Visiting Senior Research Fellowship, and also the Royal Society/Leverhulme Trust for a Senior Research Fellowship. NAMM acknowledges the support of BBSRC (grant no. 50/MMI09729) and thanks the Issac Newton Institute for Mathematical Sciences, University of Cambridge, for an EPSRC-funded Visiting Senior Research Fellowship.

\section{Appendix A}

The Gierer-Meinhardt model equations are given by

$$
u_{t}=k_{1}+k_{3} \frac{u^{2}}{v}-k_{2} u+D_{u} u_{x x}, \quad v_{t}=k_{4} u^{2}-k_{5} v+D_{v} v_{x x} \quad \text { on }[0, L],
$$

where $u$ is the activator concentration, $v$ the inhibitor concentration and $k_{1}, \ldots, k_{5}$ the positive parameters, see [10]. To be fully prescribed the equations also require boundary conditions on $u$ and $v$ at $x=0, L$ (typically zero flux). We introduce nondimensional quantities

$$
\begin{aligned}
& \bar{x}=\frac{x}{L}, \quad \bar{t}=\frac{t}{T}, \quad \bar{u}=\frac{u k_{3}}{k_{4}}, \quad \bar{v}=\frac{v k_{4}^{2} k_{1}}{k_{3}^{2}}, \\
& r=\frac{k_{1} k_{4} T}{k_{3}}, \quad \mu=T k_{2}, \quad v=T k_{5}, \quad \bar{D}_{u}=\frac{T D_{u}}{L^{2}}, \quad \bar{D}_{v}=\frac{T D_{v}}{L^{2}},
\end{aligned}
$$

where $T$ is a fixed timescale. On substituting into Eq. (A.1) these yield

$$
\bar{u}_{\bar{t}}=r\left(\frac{\bar{u}^{2}}{\bar{v}}+1\right)-\mu \bar{u}+\bar{D}_{u} \bar{u}_{\bar{x} \bar{x}}, \quad \bar{v}_{\bar{t}}=r \bar{u}^{2}-v \bar{v}+\bar{D}_{v} \bar{v}_{\bar{x} \bar{x}} \quad \text { on }[0,1] .
$$

This is the nondimensional form of the equations used in the paper (we drop the bars for notational convenience). We can see that there are fewer parameters involved in the nondimensional system, which makes analysis of the system easier. However, it should be noted that the times, lengths and concentrations in the nondimensional system are not the real values involved in the original system. In this nondimensionalisation, we could simplify the equations further by choosing an appropriate timescale $T$ (in terms of the other model parameters). However, we choose not to do this to allow us to investigate timescales involved in the emergence of patterns in reaction-diffusion systems ([25] and in progress).

\section{References}

[1] R.A. Barrio, C. Varea, J.L. Aragon, P.K. Maini, A two-dimensional numerical study of spatial pattern formation in interacting Turing systems, Bull. Math. Biol. 61 (1999) 483-505. 
[2] D.L. Benson, J.A. Sherratt, P.K. Maini, Diffusion driven instability in an inhomogeneous domain, Bull. Math. Biol. 55 (1993) $365-384$.

[3] S.S. Blair, Limb development: marginal fringe benefits, Curr. Biol. 7 (1997) R686-R690.

[4] F. Bruemmer, G. Zempel, P. Buehle, J.-C. Stein, D.F. Huesler, Retinoic acid modulates gap junctional permeability: a comparative study of dye spreading and ionic coupling in cultured cells, Exp. Cell. Res. 196 (1991) 158-163.

[5] V. Castets, E. Dulos, J. Boissonade, P. De Kepper, Experimental evidence of a sustained standing Turing-type nonequilibrium chemical pattern, Phys. Rev. Lett. 64 (1990) 2953-2956.

[6] C.N.D. Coelho, R.A. Cosher, A gradient of gap junctional communication along the anterior-posterior axis of the developing chick limb bud, Dev. Biol. 148 (1991) 529-535.

[7] R. Dillon, P.K. Maini, H.G. Othmer, Pattern formation in generalized Turing systems. I. Steady-state patterns in systems with mixed boundary conditions, J. Math. Biol. 32 (1994) 345-393.

[8] P.C. Fife, Mathematical Aspects of Reacting and Diffusing Systems, Springer, Berlin, 1979.

[9] R.J. Fleming, Y. Gu, N.A. Hukriede, Serrate-mediated activation of Notch is specifically blocked by the product of the gene fringe in the dorsal compartment of the Drosophila wing imaginal disc, Development 124 (1997) 2973-2981.

[10] A. Gierer, H. Meinhardt, A theory of biological pattern formation, Kybernetik 12 (1972) 30-39.

[11] B.C. Goodwin, S. Kaufmann, J.D. Murray, Is morphogenesis an intrinsically robust process?, J. Theor. Biol. 163 (1993)135-144.

[12] P. Grindrod, The Theory and Application of Reaction-Diffusion Equations, 2nd ed., Clarendon Press, Oxford, 1996.

[13] A. Hunding, M. Brøns, Bifurcation in a spherical reaction-diffusion system with imposed gradient, Physica D 44 (1990) $285-302$.

[14] K.D. Irvine, Fringe, Notch, and making developmental boundaries, Curr. Opin. Gen. Dev. 9 (1999) 434-441.

[15] K.D. Irvine, C. Rauskolb, Boundaries in development: formation and function, Annu. Rev. Cell Dev. Biol. 17 (2001) $189-214$.

[16] T. Klein, A. Martinez Arias, Interactions among Delta, Serrate and Fringe modulate Notch activity during Drosophila wing development, Development 125 (1998) 2951-2962.

[17] P.M. Kulesa, G.C. Cruywagen, S.R. Lubkin, P.K. Maini, J. Sneyd, M.W.J. Ferguson, J.D. Murray, On a model mechanism for the spatial patterning of teeth primordia in the alligator, J. Theor. Biol. 180 (1996) 287-296.

[18] P.K. Maini, M.R. Myerscough, Boundary-driven instability, Appl. Math. Lett. 10 (1997) 1-4.

[19] P.K. Maini, K.J. Painter, H.H.P. Chau, Spatial pattern formation in chemical and biological systems, J. Chem. Soc., Faraday Trans. 93 (1997) 3601-3610.

[20] H. Meinhardt, Cell determination boundaries as organizing regions of secondary embryonic fields, Dev. Biol. 96 (1996) $375-385$.

[21] J.D. Murray, A pre-pattern formation mechanism for animal coat markings, J. Theor. Biol. 88 (1981) 161-199.

[22] J.D. Murray, Mathematical Biology, Biomathematics Texts, vol. 19, Springer, Berlin, 1989.

[23] B.N. Nagorka, The reaction-diffusion (RD) theory of wool (hair) follicle initiation and development. II. Original secondary follicles, Aust. J. Agric. Res. 46 (1995) 357-378.

[24] H.G. Othmer, E. Pate, Scale-invariance in reaction-diffusion models of spatial pattern formation, PNAS 77 (1980) $4180-4184$.

[25] K.M. Page, Mathematical models in embryology: the selection, regulation and speed of formation of patterns, D.Phil. Thesis, University of Oxford, Oxford, 1999.

[26] V.M. Panin, V. Papayannopoulos, K.D. Irvine, Fringe modulates Notch-ligand interactions, Nature 387 (1997) 908-912.

[27] J. Schnakenberg, Simple chemical kinetics with limit cycle behaviour, J. Theor. Biol. 81 (1979) 389-400.

[28] J. Taipale, J. Keski-Oja, Growth factors in the extracellular matrix, FASEB J. 11 (1997) 51-59.

[29] T. Tabata, Genetics of morphogen gradients, Nat. Rev. Gen. 2 (2001) 620-630.

[30] D. Thomas, Artificial enzyme membranes, transport, memory and oscillatory phenomena, in: Analysis and Control of Immobilized Enzyme Systems, 1975.

[31] A.M. Turing, The chemical basis of morphogenesis, Phil. Trans. R. Soc. 237 (1952) 37-72.

[32] J.-P. Vincent, J. Briscoe, Morphogens, Curr. Biol. 11 (2001) R851-R854.

[33] L. Wolpert, Positional information and the spatial pattern of cellular differentiation, J. Theor. Biol. 25 (1969) 1-47.

[34] R.S. Cantrell, C. Cosner, The effects of spatial heterogeneity in population dynamics, J. Math. Biol. 29 (1991) $315-338$.

[35] S.W. Pacala, J. Roughgarden, Spatial heterogeneity and inter-specific competition, Theor. Popn. Biol. 21 (1982) 92-113.

[36] N. Shigesada, Spatial distribution of rapidly dispersing animals in heterogeneous environments, in: S.A. Levin, T.G. Hallam (Eds.), Lecture Notes in Biomathematics, vol. 54, Springer, Heidelberg, 1984, pp. 478-491.

[37] K. Tarumi, E. Mueller, Wavelength selection mechanism in the Gierer-Meinhardt model, Bull. Math. Biol. 51 (1989) $207-216$. 